\title{
Pulmonary Cryptococcus Infection After Mono-Chemotherapy With Gemcitabine
}

\author{
Yu-Guang Chen MD, Te-Yu Lin MD, Gen-Min Lin MD, and Jung-Chung Lin MD PhD
}

\begin{abstract}
Mono-chemotherapy with gemcitabine (difluorodeoxycytidine) is an effective cancer chemotherapy, and is often used instead of a more effective but more toxic combination chemotherapy regimen. Infection associated with gemcitabine is very rare. We present a case of Cryptococcus neoformans pneumonia that developed after 5 courses of gemcitabine for advanced bladder carcinoma. Lung biopsy and antigens demonstrated $C$. neoformans. The pneumonia resolved after antifungal therapy. Key words: gemcitabine; pneumonia; Cryptococcus neoformans. [Respir Care 2011;56(3):339-341. (C) 2011 Daedalus Enterprises]
\end{abstract}

\section{Introduction}

Fungal infections have become a worldwide healthcare problem over the last decade. The most common community-acquired pathogens in fungal pneumonia are Aspergillus species, followed by Candida species. ${ }^{1}$ Based on those analyses, pulmonary Cryptococcus infections are rare. Mono-chemotherapy with gemcitabine (difluorodeoxycytidine, brand name Gemzar) effectively treats non-smallcell lung, pancreatic, bladder, and breast cancers, and has been used in patients who do not tolerate more effective but more toxic chemotherapy agents. ${ }^{2,3}$ There have been

\footnotetext{
Yu-Guang Chen MD is affiliated with the Department of Internal Medicine, Tri-Service General Hospital, Taipei, Republic of China. Te-Yu Lin MD and Jung-Chung Lin MD are affiliated with the Division of Infectious Diseases and Tropical Medicine, Department of Internal Medicine, Tri-Service General Hospital, Taipei, Republic of China. Gen-Min Lin MD is affiliated with the Division of Cardiology, Department of Internal Medicine, Tri-Service General Hospital, National Defense Medical Center, Taipei, Republic of China, and with the Division of General Medicine, Department of Internal Medicine, Hualien Armed Forces General Hospital, Hualien, Taiwan, Republic of China.
}

The authors have disclosed no conflicts of interest.

This research was partly supported by Tri-Service General Hospital grants TSGH-C97-83 and TSGH-C97-84.

Correspondence: Jung-Chung Lin MD PhD, Division of Infectious Diseases and Tropical Medicine, Department of Internal Medicine, Tri-Service General Hospital, No. 325, Section 2, Cheng-Gong Road, Neihu 114, Taipei, Republic of China. E-mail: jclin@ndmctsgh.edu.tw.

DOI: $10.4187 /$ respcare.00652 few reports of Cryptococcus in non-hematologic malignancies treated with cytotoxic therapy. We present a case of acute Cryptococcus neoformans pneumonia that developed after mono-chemotherapy with gemcitabine for 5 weeks, in a patient with advanced bladder carcinoma.

\section{Case Report}

A 75-year-old man with type 2 diabetes mellitus and advanced urothelial carcinoma of the bladder was admitted for chemotherapy and radiotherapy in December 2006. On admission he underwent chest radiograph. No specific infiltration was observed. He received weekly gemcitabine $(1,500 \mathrm{mg})$ mono-chemotherapy for 5 weeks. At the end of that treatment period he presented with a productive cough, intermittent fever, and chills.

Physical examination found an oral temperature $38.4^{\circ} \mathrm{C}$, a heart rate 108 beats/min, a respiratory rate 26 breaths/ min, and basilar crackles over both lungs. Laboratory tests showed a white-blood-cell count of $6.1 \times 10^{9}$ cells/L, with $74 \%$ neutrophils, and C-reactive protein of $15.23 \mathrm{mg} / \mathrm{dL}$ (normal $<0.5 \mathrm{mg} / \mathrm{dL}$ ). Plain chest radiograph showed new diffuse interstitial markings, patchy consolidation, and ground-glass opacities in both lungs (Fig. 1). Chest computed tomogram revealed multiple nodules, with ill-defined consolidated patchy air spaces in both lungs, predominantly in the sub-pleural region (Fig. 2). Empirical antibiotics (piperacillin and tazobactam) were administered intravenously.

Sputum cultures showed no pathological bacteria. Tuberculosis culture, polymerase chain reaction test for Pneumocystis carinii, and test for Cytomegalovirus antigen were 


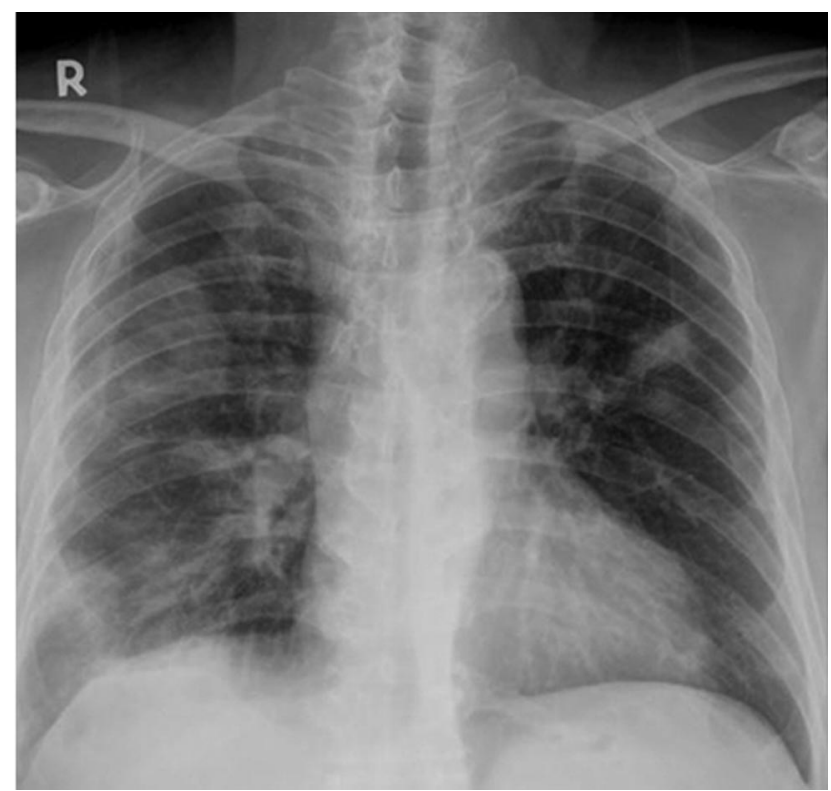

Fig. 1. Radiograph after intensive gemcitabine chemotherapy shows diffuse interstitial infiltration and patchy opacity over both lungs.

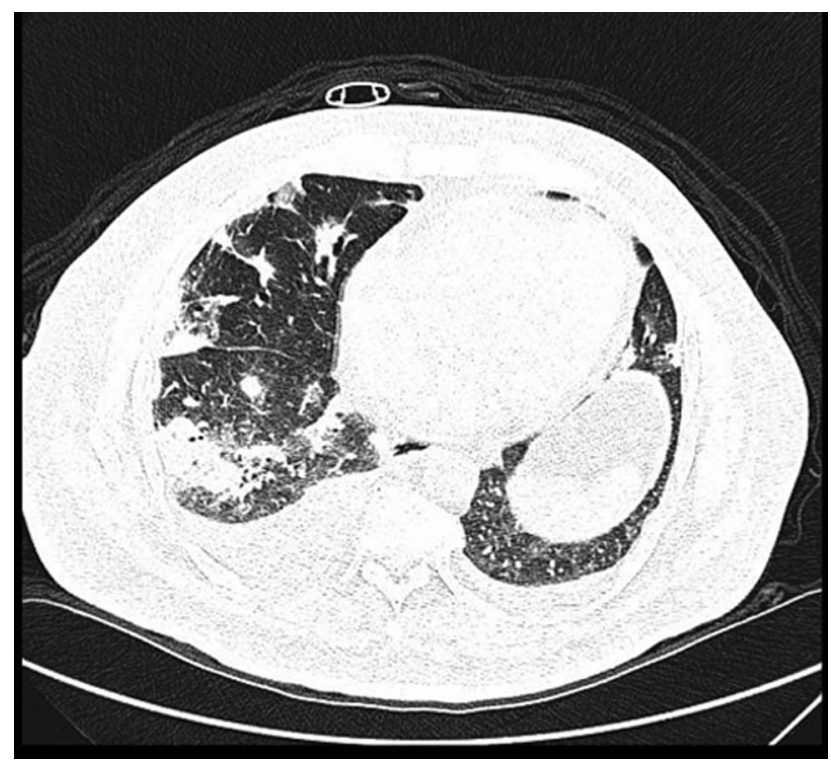

Fig. 2. Chest computed tomogram shows multiple nodules and ill-defined consolidated patches in both lungs, predominantly in the sub-pleural region, and mild to moderate pleural effusion over the right lung.

negative. The test for Cryptococcus antigen was positive, with a titer of 1:64.

Computed-tomography-guided lung biopsy was performed, and pathology confirmed pulmonary C. neoformans (Fig. 3). A subsequent lumbar puncture excluded extensive spread of $C$. neoformans. We prescribed fluconazole, $400 \mathrm{mg} / \mathrm{d}$ intravenous for one week, and orally for

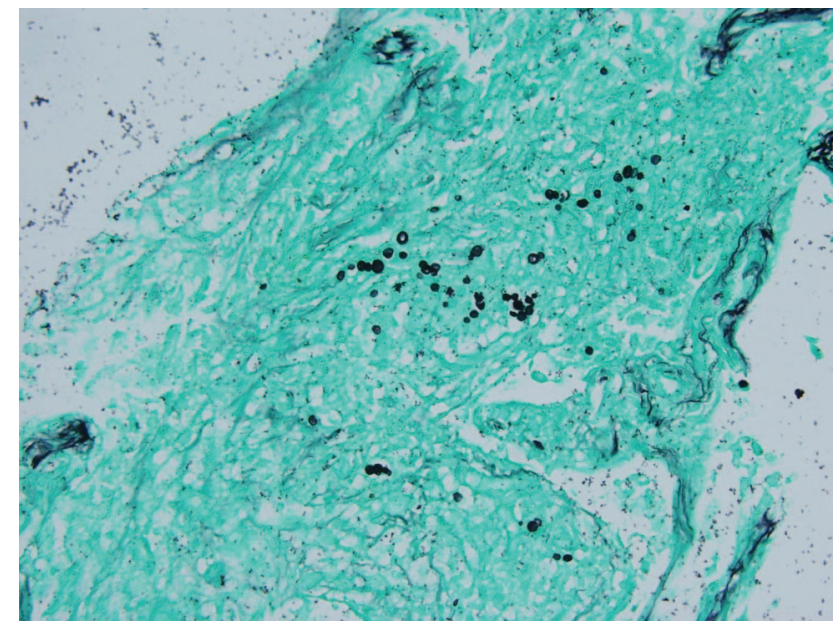

Fig. 3. Lung biopsy specimen (Grocott's methenamine silver stain, magnification 400) shows interstitial pneumonia, including thickened alveolar septa and fungal spores in the alveoli.

4 weeks. His fever gradually subsided. In a series of follow-up plain chest radiographs, the pneumonia patches completely recovered. He was free of Cryptococcus infection 6 weeks after starting fluconazole.

\section{Discussion}

Gemcitabine is a deoxycytidine anti-metabolite, antineoplastic agent. It is a pyrimidine analog, and its mechanism of action is the inhibition of DNA replication and repair in tumor cells. Gemcitabine can sequentially induce programmed cell death or apoptosis, and it is most commonly used to treat non-small-cell lung, pancreatic, bladder, and breast cancers. ${ }^{2,3}$

Gemcitabine's adverse effects include pancytopenia, nausea, vomiting, appetite loss, flu symptoms, and temporary elevated liver function. The pancytopenia can begin 10-14 days after initiating gemcitabine, and resistance to infection usually reaches its lowest point during that period. Infections associated with gemcitabine are rare.

Fungi are extremely common in the community, where they exist as free-living saprobes. They often reside on body surfaces as transient environmental colonizers, and usually do not infect immunocompetent people. Impaired $\mathrm{T}$ lymphocyte function from cancer chemotherapy and depressed neutrophil function increase the risk of fungal infection. The most common site of infection in cancer patients is the lung, and the spectrum of pulmonary infection depends upon the underlying immunologic deficiency. Several immunologic deficits can be present in one patient, which makes the patient susceptible to various opportunistic pathogens. In neutropenic patients, Gram-negative bacteria predominate early, whereas fungal infections are common if neutropenia persists. Aspergillus species are by 


\section{Pulmonary Cryptococcus Infection After Mono-Chemotherapy With Gemcitabine}

far the most common cause of fungal pneumonia in neutropenic patients, followed by Zygomycetes and Fusarium. ${ }^{4}$ Pulmonary Cryptococcus infections are very common in patients with human immunodeficiency virus, and are becoming increasingly common in patients with malignancies..$^{5,6}$

C. neoformans is an encapsulated, heterobasidiomycetous fungus found throughout the world. It is associated with the excreta of certain birds, including pigeons, canaries, and cockatoos, and with several species of eucalyptus tree. ${ }^{1}$ Clinically, the most common isolates are serotype A, typically isolated from soil and avian excreta. Based on its infective mechanism, most of these infections are acquired by inhaling environmental propagules from the soil and bird droppings.

C. neoformans infection is rare in immunocompetent patients. ${ }^{7}$ With the increasing use of immunosuppressive anti-neoplastic therapies, organ transplantation, catheter insertion, dialysis, and other invasive procedures, C. neoformans infection has become more common. The clinical manifestations of $C$. neoformans infections are variable and range from asymptomatic colonization to life-threatening disease. ${ }^{1,8,9}$ Cryptococcal meningitis is the most frequent manifestation, and pulmonary cryptococcal infection is the second most frequent. Patients may present with evidence of acute infection, such as fever, chest pain, cough, weight loss, and sputum production. In a severely immunocompromised host, cryptococcal pneumonia can rapidly progress to acute respiratory distress syndrome. ${ }^{1,8}$ Patients at risk for cryptococcal infection should avoid high-risk environments.

Pulmonary cryptococcosis can manifest as acute or nonacute pneumonia. Acute cryptococcal pneumonia is rare, sometimes corresponds with symptomatic primary infection, and can be complicated by severe acute respiratory distress syndrome. Non-acute cryptococcal pneumonia is more common, and usually corresponds with reactivation, and is often associated with meningitis or other body localizations. ${ }^{10}$ Most $C$. neoformans infections are associated with impaired cell-mediated immunity. ${ }^{11} \mathrm{Hu}-$ moral immunity also contributes to protection against C. neoformans. ${ }^{12}$

As a consequence, isolation of $C$. neoformans in a respiratory specimen warrants the physician's attention. In some cases a $C$. neoformans isolate can indicate a pulmonary infection, either alone or in association with a disseminated disease. In other cases it may indicate an asymptomatic carrier. ${ }^{13}$ It was clear in our patient that sputum fungal culture and the pellets from bronchoalveolar lavage were negative for the possibility of pulmonary Cryptococcus reactivation and that acute pulmonary cryptococcosis may have resulted from a massive environmental exposure to soil contaminated by bird droppings. These findings suggested less of a possibility of infection of the upper airway and lower airway colonization than acute infection.

We strongly suspect that the $C$. neoformans infection in our patient was community-acquired, and we emphasize that the environment may play a role in Cryptococcus infection in cancer patients, even those receiving less toxic chemotherapy. This is a very important lesson in the care and management of patients on chemotherapy.

\section{REFERENCES}

1. Duperval R, Hermans PE, Brewer NS, Roberts GD. Cryptococcosis, with emphasis on the significance of isolation of Cryptococcus neoformans from the respiratory tract. Chest 1977;72(1):13-19.

2. Bramhall SR, Schulz J, Nemunaitis J, Brown PD, Baillet M, Buckels JA. A double-blind placebo-controlled, randomised study comparing gemcitabine and marimastat with gemcitabine and placebo as first line therapy in patients with advanced pancreatic cancer. Br J Cancer 2002;87(2):161-167.

3. Xie DR, Liang HL, Yang Q, Guo SS, Jiang ZM. [Meta-analysis on gemcitabine of fixed-dose rate infusion plus oxaliplatin as first-line therapy for advanced pancreatic cancer.] Ai Zheng 2007;26(8):895899. Article in Chinese.

4. Pagano L, Fianchi L, Leone G. Fungal pneumonia due to molds in patients with hematological malignancies. J Chemother 2006;18(4): 339-352.

5. Friedman GD, Fessel J, Udaltsova N, Hurley LB. Smoking and cryptococcosis in AIDS patients. Mycoses 2006;49(1):68-69.

6. Friedman GD, Jeffrey FW, Udaltsova NV, Hurley LB. Cryptococcosis: the 1981-2000 epidemic. Mycoses 2005;48(2):122-125.

7. Aberg JA, Mundy LM, Powderly WG. Pulmonary cryptococcosis in patients without HIV infection. Chest 1999;115(3):734-740.

8. Cameron ML, Bartlett JA, Gallis HA, Waskin HA. Manifestations of pulmonary cryptococcosis in patients with acquired immunodeficiency syndrome. Rev Infect Dis 1991;13(1):64-67.

9. Perfect JR, Casadevall A. Cryptococcosis. Infect Dis Clin North Am 2002;16(4):837-842.

10. Kerkering TM, Duma RJ, Shadomy S. The evolution of pulmonary cryptococcosis: clinical implications from a study of 41 patients with and without compromising host factors. Ann Intern Med 1981;94(5): 611-616.

11. Schop J. Protective immunity against Cryptococcus neoformans infection. Mcgill J Med 2007;10(1):35-43.

12. Vecchiarelli A, Casadevall A. Antibody-mediated effects against Cryptococcus neoformans: evidence for interdependency and collaboration between humoral and cellular immunity. Res Immunol 1998; 149(4-5):321-333.

13. Rozenbaum R, Goncalves AJ. Clinical epidemiological study of 171 cases of cryptococcosis. Clin Infect Dis 1994;18(3):369-380. 